\title{
Evidence of Processing Non-Idealities in 4H-SiC Integrated Circuits Fabricated With Two Levels of Metal Interconnect
}

David J. Spry 1 , Philip G. Neudeck¹, Liangyu Chen², Laura J. Evans ${ }^{1}$, Dorothy Lukco 3 , Carl W. Chang ${ }^{3}$, Glenn M. Beheim¹,

1) NASA Glenn Research Center, 21000 Brookpark Rd., Cleveland, OH 44135, USA

2) Ohio Aerospace Institute, 21000 Brookpark Rd. 77-1, Cleveland, OH 44135, USA

3) Vantage Partners LLC, 21000 Brookpark Rd. MS 77-1, Cleveland, OH 44135, USA

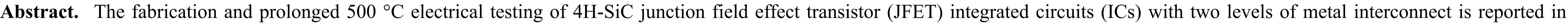

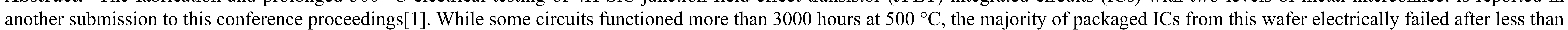

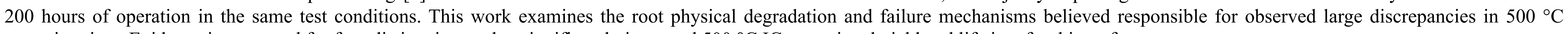
operating time. Evidence is presented for four distinct issues that significantly impacted $500{ }^{\circ} \mathrm{C}$ IC operational yield and lifetime for this wafer.
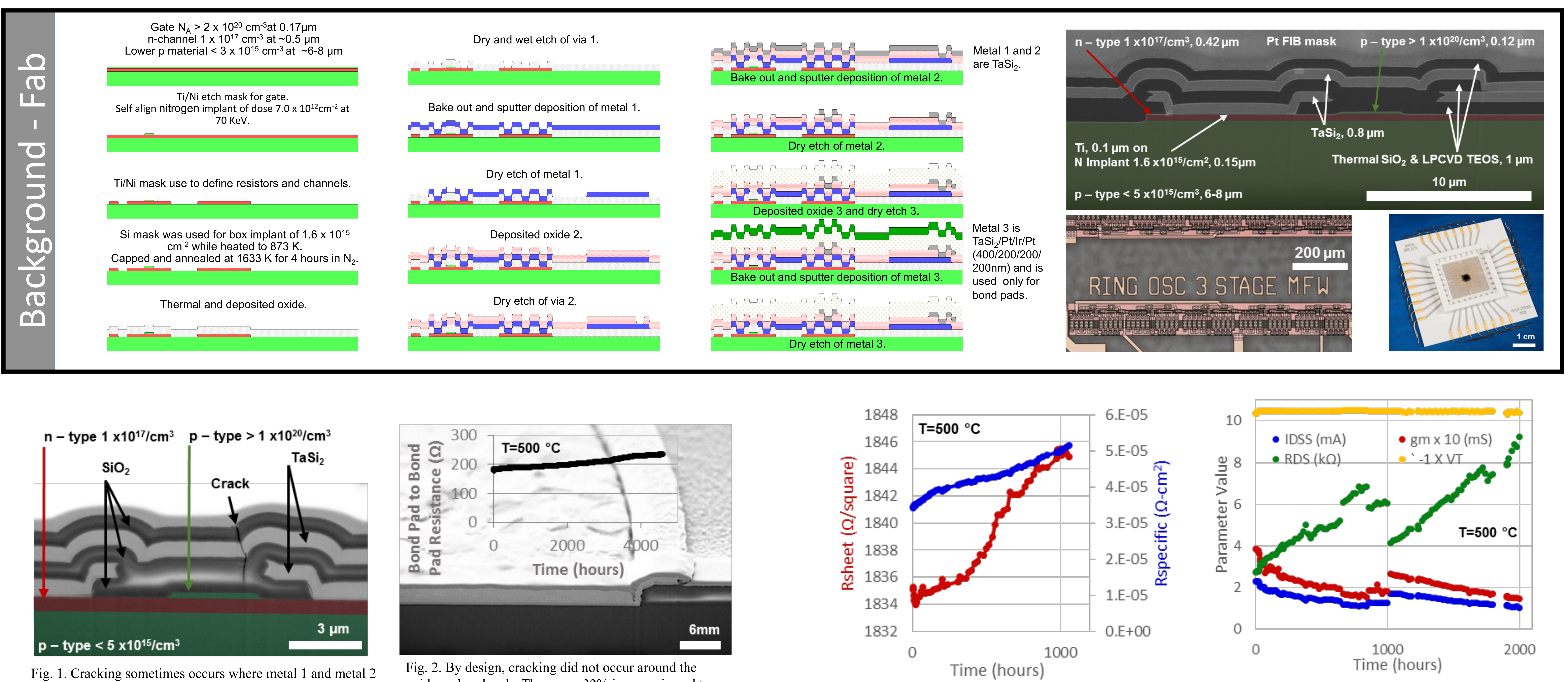

Fig. 1. Cracking so
over laps the gate.

Crack Formation Induced Fail-Open of Top Metal Interconnect. The focused ion beam (FIB) cross-section shown in Fig.1, illustrates an example of dielectric and metal cracks that caused topmost metal conductive interconnect traces to electrically fail open. Devices close to the wafer center (roughly $<1 \mathrm{~cm}$ ) did not exhibit this problem as the misalignment in this region was insufficient to result in such overlap/distortion. Misalignment and cracking grew more severe with increasing radial distance from the wafer center, consistent with rotational misalignment of the bottom metal mask pattern to the substrate n-mesa pattern during fabrication. Such cracks are not observed on other kinds of topology, such as where the lower $\mathrm{TaSi}_{2}$ trace edge ends over the flat $\mathrm{SiC}$ region on the left side of Fig. 1. Cracking is also not observed on the large bond pad edge cross-section shown in Fig. 2. The measured resistance vs. time between adjacent connected bond pads plotted in Fig. 2 demonstrates desired minimal change in conduction properties (in part due to absence of crack features) over 4800 hours of $500{ }^{\circ} \mathrm{C}$ electrical testing time. Since it is related to misalignment, this cracking problem should be solvable by improved alignment and/or design rules with larger misalignment tolerances so as to prevent overlapping of lower metal edges with mesa features.

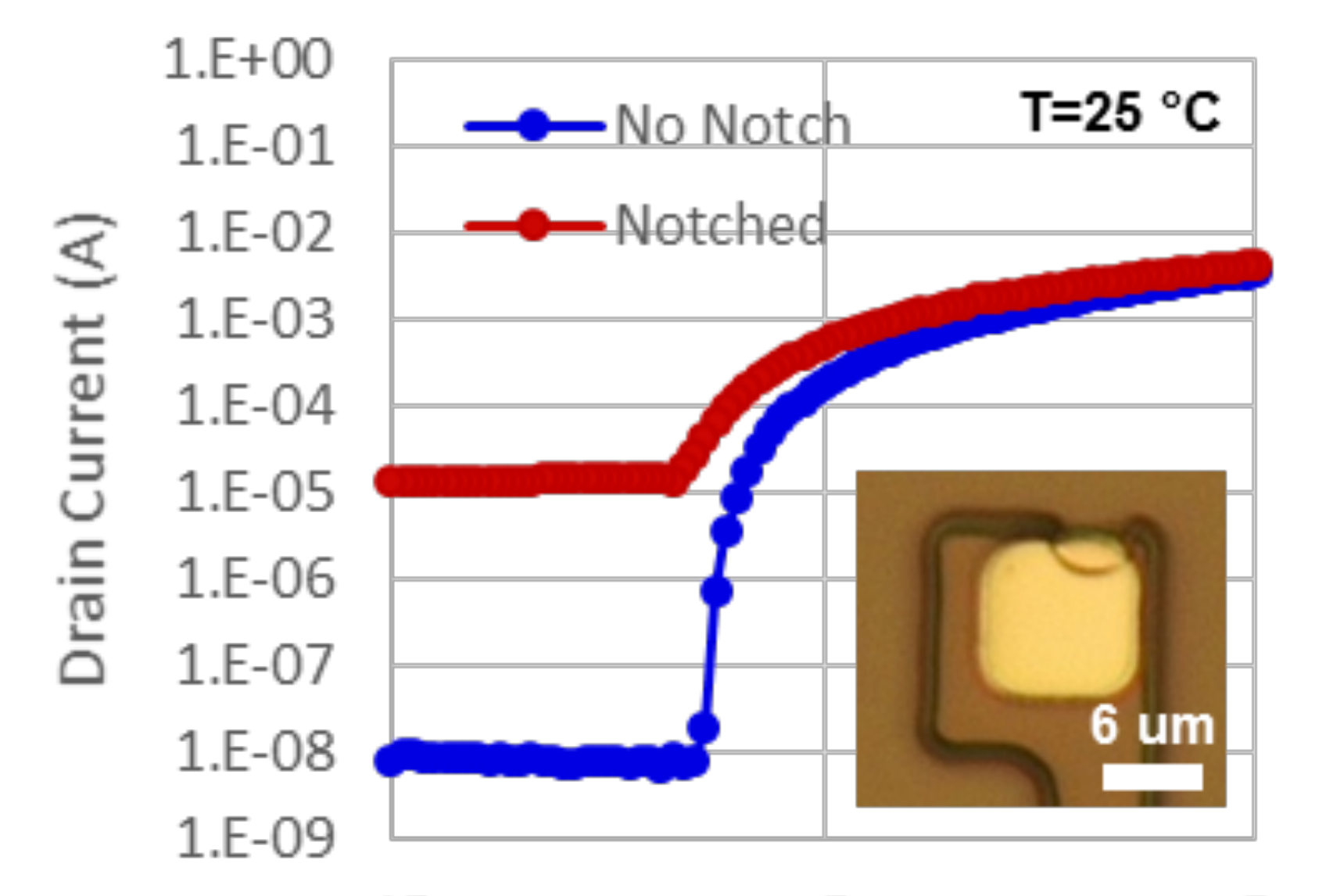

Gate Voltage (V) Fig. 3. JFET turn off leakage with and without a notch
in the p-gate mesa.

Gate-to-Channel Leakage/Short From Notching of Gate Etch Mask Metal. These notches arose from non-uniform oxidation/peeling along the edges of the gate etch mask during a prolonged unplanned storage of the sample in room air. When the second dry (n-mesa) etch was conducted, the notched regions did not sufficiently protect the $\mathrm{p}^{+} \mathrm{SiC}$ from removal, which then exposed underlying $n$-mesa regions in areas where contacts to $\mathrm{p}^{+}$gates were subsequently deposited. Fig. 3 inset shows where a $\mathrm{p}^{+}$gate contact overlaps such a notch. This allows the deposited metal $\mathrm{p}^{+}$gate contact to also form a parasitic electrical contact to the underlying exposed n-channel epilayer, including along the vertical etched sidewall of the n-epilayer. While not a complete short circuit at $\mathrm{T}=25^{\circ} \mathrm{C}$, turn-off characteristics of "notched" JFETs are undesirably orders of magnitude larger than notch-free devices (Fig. 3). While not directly measured at high $\mathrm{T}$, the leakage mechanisms of metal-semiconductor contacts/diodes are known to increase with anneal time and exponentially with $\mathrm{T}$ [2]. Therefore, this parasitic leakage is surmised to contribute to rapid failure of circuits at $500{ }^{\circ} \mathrm{C}$. The degree of notching (and thus the degree of leakage) varied across the wafer. Very few devices with more than 10 gate fingers were free of notches. Notches have not been observed in any process runs before and after this wafer whose processing time between the gate and mesa etches have been a week or less, and were shipped to vendors in vacuum boxes.

Summary. Evidence for at least four kinds of device non-idealities (cracking, notching, device resistance degradation, and mobile ions) found on a $4 \mathrm{H}-\mathrm{SiC}$ JFET IC wafer process for $500{ }^{\circ} \mathrm{C}$ operation have been described. Elimination/mitigation of the three most rapid failure mechanisms (cracking, notching, and mobile ions) is believed possible in future wafers with relatively straightforward process improvements.
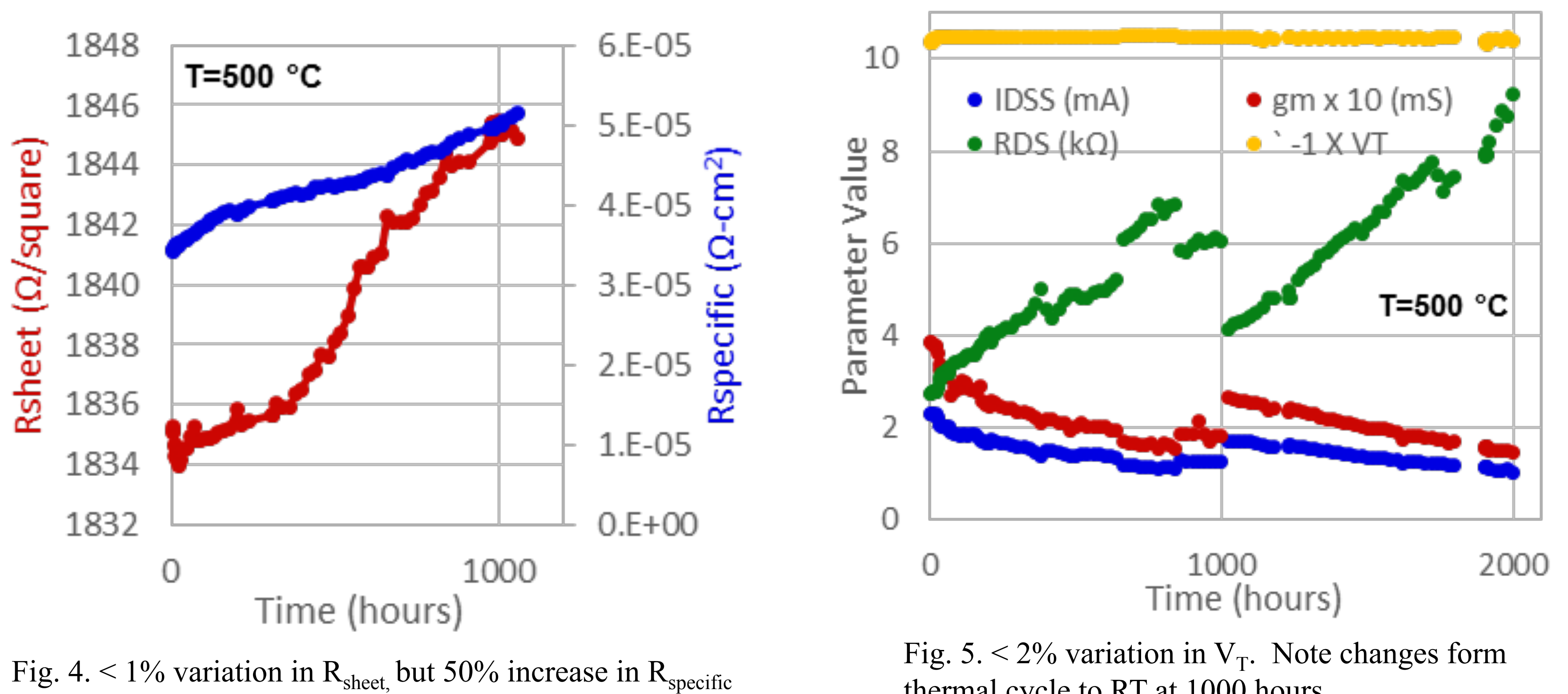

Device Resistance Degradation. Fig. 4 plots sheet resistance $\left(\mathrm{R}_{\text {Sheet }}\right)$ and specific contact resistance $\left(\mathrm{R}_{\text {Specific }}\right)$ extracted from prolonged measurement of a packaged $500{ }^{\circ} \mathrm{C}$ transmission line method (TLM) test device of the source/drain implant for over 1000 hours. $\mathrm{R}_{\text {Sheet }}$ changed less than $1 \%$ while $\mathrm{R}_{\text {Specific }}$ changed $50 \%$. This suggests a degradation in the contact area or metal while the high-dose $\mathrm{SiC}$ implanted sheet resistance is stable. The $500{ }^{\circ} \mathrm{C}$ testing time evolution of transistor parameters for a JFET in a NOT circuit are shown in Fig. 5. The threshold voltage $\left(\mathrm{V}_{\mathrm{T}}\right)$ changes less than $2 \%$ over 2000 hours. Transconductance $\left(\mathrm{g}_{\mathrm{m}}\right)$ and the on-state current $\left(\mathrm{I}_{\mathrm{DSS}}\right.$ ) decrease with time at temperature while on-state resistance $R_{D S}$ increases, which is qualitatively consistent with the Fig. 4 trend. Data discontinuity shown in Fig. 5 at 1000 hours corresponds to a brief temperature cycle to $25^{\circ} \mathrm{C}$ and back. Most of the drift in $\mathrm{R}_{\mathrm{DS}}$ recovered during this cycle. It is important to note that the recovery of $\mathrm{R}_{\mathrm{DS}}$ seems inconsistent with contact oxidation or metal trace failure mechanisms that would be irrecoverable. Delamination of the contact area has not been seen in focused ion beam (FIB) cut cross-section images of similar devices on the wafer. Therefore, the root physical cause (or causes) of this degradation remains under investigation at this time.
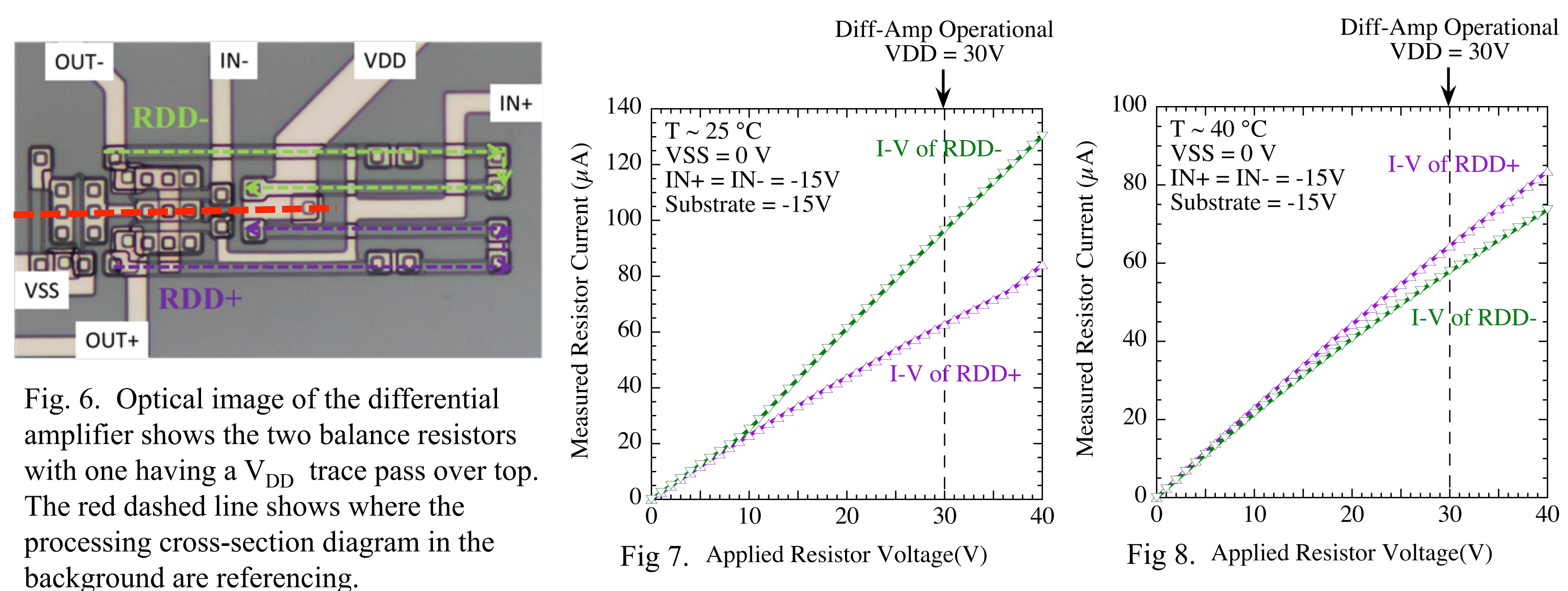

Parasitic Circuit Imbalance Induced by Mobile Ion Contamination. Differential amplifier circuits rely on well-balanced circuit legs (i.e., transistors and/or resistors) to operate. Resistive load differential amplifier ICs (including 2-stage op-amps which were also used as sense-amps for test memory ICs) were fabricated and electrically verified as operational at room temperature with expected gains. However, on all ICs custom-packaged for high temperature testing, these differential-based amplifiers all rapidly failed as they were heated past $200{ }^{\circ} \mathrm{C}$. A packaged diff-amp (Fig. 6) underwent bias-temperature stress measurements while directly measuring "balanced" resistors RDD+ and RDD- (highlighted with colored dashed lines) IV characteristics. Fig. 7 shows an example of the resistor IV imbalance measured after $+30 \mathrm{~V} 200^{\circ} \mathrm{C}$ positive bias stress of the VDD metal trace, while Fig. 8 shows that the imbalance is mostly eliminated following a subsequent $-18 \mathrm{~V} 300{ }^{\circ} \mathrm{C}$ stress applied to the VDD metal trace. This reversible bias-temperature-time stress behavior is consistent with reversible mobile ion contamination phenomenon well-known to be detrimental to metal-oxide-semiconductor (MOS) devices [3]. Subsequent surface analysis detected sodium $(\mathrm{Na})$ on the surface of wafer samples.

Acknowledgement: K. Moses, J. Gonzalez, A. Avishai, M. Mrdenovich, R. Meredith, R. Buttler, L. Matus, G. Hunter, and the NASA Transformative Aeronautics Concept Program.

References

[1] D. J. Spry et al., submitted to ICSCRM 2015

[2] Raymond T. Tung, The physics and chemistry of the Schottky barrier height, Applied Physics Reviews 1, $011304(2014)$

[3] "Built-in reliability Through Sodium Elimination", J. Chinn, Y.-S. Ho and M. Chiang [Siliconix], IEEE Intl Reliability Phys Symp 1994 p. 249 